\title{
A New Method of Multiattribute Decision-Making Based on Interval-Valued Hesitant Fuzzy Soft Sets and Its Application
}

\author{
Yan Yang, ${ }^{1}$ Lei Lang, ${ }^{1}$ Liuli $\mathbf{L u},{ }^{2}$ and Yangmei Sun ${ }^{1}$ \\ ${ }^{1}$ College of Science, Southwest Petroleum University, Chengdu 610500, China \\ ${ }^{2}$ School of Information and Computation Science, Chengdu Technological University, Chengdu 610500, China \\ Correspondence should be addressed to Yan Yang; 42188780@qq.com
}

Received 11 April 2017; Revised 9 June 2017; Accepted 19 June 2017; Published 30 July 2017

Academic Editor: Franck Massa

Copyright (c) 2017 Yan Yang et al. This is an open access article distributed under the Creative Commons Attribution License, which permits unrestricted use, distribution, and reproduction in any medium, provided the original work is properly cited.

Combining interval-valued hesitant fuzzy soft sets (IVHFSSs) and a new comparative law, we propose a new method, which can effectively solve multiattribute decision-making (MADM) problems. Firstly, a characteristic function of two interval values and a new comparative law of interval-valued hesitant fuzzy elements (IVHFEs) based on the possibility degree are proposed. Then, we define two important definitions of IVHFSSs including the interval-valued hesitant fuzzy soft quasi subset and soft quasi equal based on the new comparative law. Finally, an algorithm is presented to solve MADM problems. We also use the method proposed in this paper to evaluate the importance of major components of the well drilling mud pump.

\section{Introduction}

In 1965, Zadeh [1] introduced the notion of fuzzy set. After that, a variety of extended fuzzy set theories have been proposed such as intuitionistic fuzzy set [2], interval-valued intuitionistic fuzzy set [3], hesitant fuzzy set $[4,5]$, and interval-valued hesitant fuzzy set [6]. In 1999, Molodtsov [7] firstly proposed soft set theory, which has been widely used in many different areas such as decision analysis [8] and military research [9]. With diversity and complexity of practical problems, the original theories are inapplicable to solve these problems. Maji et al. [10] firstly explored fuzzy soft set, which is a more general notion combining fuzzy set and soft set. Some extensions of fuzzy soft set theory have also been presented such as interval-valued fuzzy soft set [11], intuitionistic fuzzy soft set [12], and interval-valued intuitionistic fuzzy soft set [13]. In 2015, Peng and Yang [14] proposed interval-valued hesitant fuzzy soft set (IVHFSS) combining interval-valued hesitant fuzzy set and soft set.

In the aspect of the research works of MADM problems, Roy and Maji [15] presented a method according to a comparison table from fuzzy soft sets. Kong et al. [16] modified Roy and Maji's algorithm to give a novel one, which is based on the comparison of choice values of different objects. Feng et al.
[17] proposed a new approach by using level soft sets to solve MADM problems based on fuzzy soft sets. Yang et al. [18] used Feng et al.'s algorithm to solve MADM problems based on multiple fuzzy soft sets. Xu and Zhang [19] developed a novel approach by TOPSIS with incomplete weight information for solving hesitant fuzzy MADM problems. Wang et al. [20] proposed a MADM method based on hesitant fuzzy soft sets. Wang et al. [21] proposed a MADM approach based on the aggregation operator to aggregate hesitant fuzzy soft information. Peng and Yang [14], by means of TOPSIS and the maximizing deviation method, presented a MADM method based on interval-valued hesitant fuzzy soft sets.

The method proposed by Peng and Yang is very effective and allows a decision-maker to quantify his opinion with an interval value within $[0,1]$, since it may be difficult for a decision-maker to exactly quantify his opinion with a crisp number to an attribute due to the insufficiency of available information in practical MADM problems. However, there are two problems with this method. One is that it only considers opinion of a decision-maker and the other is that the comparative law based on the score function is not accurate when it is used to compare two IVHFEs in dealing with MADM problems. Based on the above analysis, we improve Peng and Yang's method to propose a new one 
of MADM methods based on IVHFSSs, which not only synthetically considers opinions of many decision-makers but also increases the accuracy of the comparison between two IVHFEs.

The remainder of this paper is organized as follows. In Section 2, some basic definitions including interval value, possibility degree, interval-valued hesitant fuzzy set, and interval-valued hesitant fuzzy soft set are briefly reviewed. In Section 3, a characteristic function of two interval values and a comparative law of IVHFEs based on the possibility degree are proposed. In Section 4, we define the intervalvalued hesitant fuzzy soft quasi subset and soft quasi equal based on the new comparative law presented in Section 3. In Section 5, a MADM method based on IVHFSSs is presented. In Section 6, we apply this method to evaluate the importance of major components of the well drilling mud pump. Analysis and discussion are presented in Section 7. The paper is concluded in Section 8.

\section{Preliminaries}

In this section, we mainly review some definitions including interval value, possibility degree of interval value, intervalvalued hesitant fuzzy set, and interval-valued hesitant fuzzy soft set. Let $U$ be an initial universe set of objects and let $E$ be the set of attributes in relation to objects in $U ; A \subseteq E$.

Definition 1 (see [22]). Let $r=\left[r^{L}, r^{U}\right]=\left\{x \mid 0 \leq r^{L} \leq x \leq\right.$ $\left.r^{U}\right\}$; then $r$ is called a nonnegative interval value. Particularly, $r$ is a nonnegative real number, if $r^{L}=r^{U}$.

The comparison of two interval values can be roughly divided into two categories: one is deterministic ordering method [23] and the other is possibility degree method [24]. In this paper, we select the latter method.

Definition 2 (see [24]). Let $r_{1}=\left[{r_{1}}^{L}, r_{1}{ }^{U}\right]$ and $r_{2}=\left[r_{2}{ }^{L}, r_{2}{ }^{U}\right]$ be two interval values, and $\lambda \geq 0$; then

(1) $r_{1}+r_{2}=\left[r_{1}^{L}+r_{2}^{L}, r_{1}^{U}+r_{2}^{U}\right]$;

(2) $\lambda r_{1}=\left[\lambda r_{1}^{L}, \lambda r_{1}^{U}\right]$; particularly, $\lambda r_{1}=0$, if $\lambda=0$.

Definition 3 (see [24]). Let $r_{1}=\left[r_{1}{ }^{L}, r_{1}{ }^{U}\right]$ and $r_{2}=\left[r_{2}{ }^{L}, r_{2}{ }^{U}\right]$ be two interval values; then the possibility degree of $r_{1} \geq r_{2}$ is defined as follows:

$$
\begin{aligned}
& P\left(r_{1} \geq r_{2}\right) \\
& \quad=\max \left\{1-\max \left(\frac{r_{2}{ }^{U}-r_{1}{ }^{L}}{\left(r_{1}{ }^{U}-r_{1}{ }^{L}\right)+\left(r_{2}{ }^{U}-r_{2}{ }^{L}\right)}, 0\right), 0\right\} .
\end{aligned}
$$

Definition 4 (see [25]). Let $r_{1}=\left[r_{1}{ }^{L}, r_{1}{ }^{U}\right]$ and $r_{2}=\left[r_{2}{ }^{L}, r_{2}{ }^{U}\right]$ :

(1) If $P\left(r_{1} \geq r_{2}\right)=0.5$, then $a$ is quasi-equal to $b$ denoted by $r_{1} \approx r_{2}$.

(2) If $P\left(r_{1} \geq r_{2}\right)>0.5$, then $a$ is more than $b$ denoted by $r_{1}>r_{2}$.

(3) If $P\left(r_{1} \geq r_{2}\right)<0.5$, then $a$ is less than $b$ denoted by $r_{1} \prec r_{2}$.
Definition 5 (see $[4,5])$. Let $X$ be a fixed set. A hesitant fuzzy set on $X$ is in terms of a function that when applied to $X$ returns a subset of $[0,1]$, which can be represented as the following mathematical symbol:

$$
H=\left\{\frac{h_{H}(x)}{x} \mid x \in X\right\},
$$

where $h_{H}(x)$ is a set of values in $[0,1]$, denoting the possible membership degrees of the element $x \in X$ to the set $H$. For convenience, we call $h_{H}(x)$ a hesitant fuzzy element.

Definition 6 (see [6]). Let $X$ be a reference set, and let $D[0,1]$ be the set of all closed subintervals of $[0,1]$. An intervalvalued hesitant fuzzy set (IVHFS) on $X$ is

$$
\bar{H}=\left\{\frac{h_{\bar{H}}(x)}{x} \mid x \in X\right\},
$$

where $h_{\bar{H}}(x): X \rightarrow D[0,1]$ denotes all possible intervalvalued membership degrees of the element $x \in X$ to the set $\bar{H}$. For convenience, we call $h_{\bar{H}}(x)$ an interval-valued hesitant fuzzy element (IVHFE), which reads $h_{\bar{H}}(x)=\left\{r \mid r \in h_{\bar{H}}(x)\right\}$.

Definition 7 (see [6]). Let $h_{\bar{H}_{1}}(x)$ and $h_{\bar{H}_{2}}(x)$ be two IVHFEs, where $h_{\bar{H}_{1}}(x)=\left\{r_{1}=\left[r_{1}{ }^{L}, r_{1}{ }^{U}\right] \mid r_{1} \in h_{\bar{H}_{1}}(x)\right\}$ and $h_{\bar{H}_{2}}(x)=$ $\left\{r_{2}=\left[r_{2}{ }^{L}, r_{2}{ }^{U}\right] \mid r_{2} \in h_{\bar{H}_{2}}(x)\right\}$; then the operational laws of IVHFEs are defined as follows:

$$
\text { (1) } \begin{aligned}
& \lambda\left(h_{\bar{H}_{1}}(x)\right)=\left\{\left[1-\left(1-r_{1}{ }^{L}\right)^{\lambda}, 1-\left(1-r_{1}{ }^{U}\right)^{\lambda}\right] \mid r_{1} \in\right. \\
& \left.h_{\bar{H}_{1}}(x)\right\}, \lambda>0 . \\
\text { (2) } h_{\bar{H}_{1}}(x) \cap h_{\bar{H}_{2}}(x)=\left\{\left[\min \left(r_{1}{ }^{L}, r_{2}{ }^{L}\right), \min \left(r_{1}{ }^{U}, r_{2}{ }^{U}\right)\right] \mid\right. & \\
& \left.r_{1} \in h_{\bar{H}_{1}}(x), r_{2} \in h_{\bar{H}_{2}}(x)\right\} . \\
\text { (3) } h_{\bar{H}_{1}}(x) \cup h_{\bar{H}_{2}}(x)=\left\{\left[\max \left(r_{1}{ }^{L}, r_{2}{ }^{L}\right), \max \left(r_{1}{ }^{U}, r_{2}{ }^{U}\right)\right] \mid\right. & \\
& \left.r_{1} \in h_{\bar{H}_{1}}(x), r_{2} \in h_{\bar{H}_{2}}(x)\right\} .
\end{aligned}
$$

Definition 8 (see [7]). Let $P(U)$ be the set of all subsets of $U$. A pair $(\bar{F}, A)$ is called a soft set over $U$, where $\bar{F}$ is a mapping given by $\bar{F}: A \rightarrow P(U)$.

Definition 9 (see [14]). Let $\operatorname{IVHF}(U)$ be the set of all intervalvalued hesitant fuzzy subsets of $U$. A pair $(\bar{F}, A)$ is called an interval-valued hesitant fuzzy soft set (IVHFSS) over $U$, where $\bar{F}$ is a mapping given by $\bar{F}: A \rightarrow \operatorname{IVHF}(U)$.

In [14], some properties of IVHFSSs were given. We give another three properties as follows.

Property 10. Given an IVHFSS $(\bar{F}, A)$ over $U$,

(1) if $A=\{e\}$, then $(\bar{F}, A)$ is also an interval-valued hesitant fuzzy set,

(2) if there is only one interval value of $h_{\bar{F}(e)}(u)$ for all $e \epsilon$ $A$ and $u \in U$, then $(\bar{F}, A)$ is also an interval-valued fuzzy soft set,

(3) if the upper and lower boundaries of each interval value of $h_{\bar{F}(e)}(u)$ are equal for all $e \in A$ and $u \in U$, then $(\bar{F}, A)$ is also a hesitant fuzzy soft set. 
Definition 11 (see [14]). The union operation on two IVHFSSs $(\bar{F}, A)$ and $(\bar{G}, B)$ over $U$ is an IVHFSS $(\bar{H}, C)$, where $C=$ $A \cup B$ and, for all $e \in C$,

$$
\bar{H}(e)= \begin{cases}\bar{F}(e), & \text { if } e \in A-B, \\ \bar{G}(e), & \text { if } e \in B-A, \\ \bar{F}(e) \cup \bar{G}(e), & \text { if } e \in A \cap B .\end{cases}
$$

We write $(\bar{F}, A) \bar{\cup}(\bar{G}, B)=(\bar{H}, C)$.

Definition 12 (see [14]). The intersection operation on two IVHFSSs $(\bar{F}, A)$ and $(\bar{G}, B)$ with $A \cap B \neq \Phi$ over $U$ is an IVHFSS $(\bar{H}, C)$, where $C=A \cap B$ and, for all $e \in C$, $\bar{H}(e)=\bar{F}(e) \cap \bar{G}(e)$. We write $(\bar{F}, A) \bar{\cap}(\bar{G}, B)=(\bar{H}, C)$.

\section{A New Comparative Law of Interval-Valued Hesitant Fuzzy Elements Based on the Possibility Degree}

In this section, a new comparative law of IVHFEs will be proposed which can overcome some defects of the comparative law based on the score function.

For convenience, let $l\left(h_{\bar{H}}(x)\right)$ denote the length of $h_{\bar{H}}(x)$ and let $h_{\bar{H}}{ }^{\sigma(j)}(x)$ denote the $j$ th maximum interval value in $h_{\bar{H}}(x) . l_{\max }$ is the maximum value of $l\left(h_{\bar{H}_{1}}(x)\right)$ and $l\left(h_{\bar{H}_{2}}(x)\right)$.

Definition 13 (see [6]). The score function of $h_{\bar{H}}(x)$ is defined as follows:

$$
S\left(h_{\bar{H}}(x)\right)=\frac{1}{l\left(h_{\vec{H}}(x)\right)} \sum_{r \in h_{\bar{H}}(x)} r .
$$

Obviously, $S\left(h_{\bar{H}}(x)\right)$ is still an interval value. Given two IVHFEs $h_{\bar{H}_{1}}(x)$ and $h_{\bar{H}_{2}}(x)$,

(1) if $S\left(h_{\bar{H}_{1}}(x)\right) \approx S\left(h_{\bar{H}_{2}}(x)\right)$, then $h_{\bar{H}_{1}}(x) \approx h_{\bar{H}_{2}}(x)$;

(2) if $S\left(h_{\bar{H}_{1}}(x)\right)>S\left(h_{\bar{H}_{2}}(x)\right)$, then $h_{\bar{H}_{1}}(x)>h_{\bar{H}_{2}}(x)$;

(3) if $S\left(h_{\bar{H}_{1}}(x)\right) \prec S\left(h_{\bar{H}_{2}}(x)\right)$, then $h_{\bar{H}_{1}}(x) \prec h_{\bar{H}_{2}}(x)$.

Example 14. There are $h_{\bar{H}_{1}}(x)=\{[0.3,0.4],[0.4,0.5],[0.5$, $0.6]\}$ and $h_{\bar{H}_{2}}(x)=\{[0.3,0.4],[0.5,0.6]\}$. Obviously, $h_{\bar{H}_{1}}(x)>$ $h_{\bar{H}_{2}}(x)$. However, by Definition 13 , we have $S\left(h_{\bar{H}_{1}}(x)\right) \approx$ $S\left(h_{\bar{H}_{2}}(x)\right)$; thus $h_{\bar{H}_{1}}(x) \approx h_{\bar{H}_{2}}(x)$.

Given two IVHFEs $h_{\bar{H}_{1}}(x)$ and $h_{\bar{H}_{2}}(x)$, usually, $l\left(h_{\bar{H}_{1}}(x)\right) \neq$ $l\left(h_{\bar{H}_{2}}(x)\right)$. However, the comparative law used in Example 14 ignores this important point. In fact, according to the attitude of decision-makers, there are three different ways to make them equal in length by adding some suitable interval values. When the decision-makers are optimistic, we make them equal in length by adding the maximum interval value. Analogously, we add the minimum interval value when the decision-makers are pessimistic. The decision-makers remain neutral; we add the average of the maximum interval value and the minimum interval value. In this paper, if $l\left(h_{\bar{H}_{1}}(x)\right) \neq l\left(h_{\bar{H}_{2}}(x)\right)$, without loss of generality, we take the pessimistic principle to make $h_{\bar{H}_{1}}(x)$ and $h_{\bar{H}_{2}}(x)$ equal in length such that $l\left(h_{\bar{H}_{1}}(x)\right)=l\left(h_{\bar{H}_{2}}(x)\right)=l_{\max }$.

Example 15. Two IVHFEs are given in Example 14. Obviously, $l\left(h_{\bar{H}_{1}}(x)\right) \neq l\left(h_{\bar{H}_{2}}(x)\right)$. Since $([0.3,0.4] \succeq[0.5,0.6])=0$, $[0.3,0.4] \prec[0.5,0.6]$. Based on the pessimistic principle, we can extend $h_{\bar{H}_{2}}(x)=\{[0.3,0.4],[0.3,0.4],[0.5,0.6]\}$ such that $l\left(h_{\bar{H}_{1}}(x)\right)=l\left(h_{\bar{H}_{2}}(x)\right)=3$.

In 2014, Zhou [9] proposed a characteristic function of two real numbers. Based on this, we give a characteristic function of two interval values.

Definition 16. Assume that $r_{1}=\left[r_{1}{ }^{L}, r_{1}{ }^{U}\right]$ and $r_{2}=\left[r_{2}{ }^{L}, r_{2}{ }^{U}\right]$; then the characteristic function $f\left(r_{1}, r_{2}\right)$ is defined as follows:

$$
f\left(r_{1}, r_{2}\right)= \begin{cases}1, & \text { if } r_{1}>r_{2} \\ 0.5, & \text { if } r_{1} \approx r_{2} \\ 0, & \text { if } r_{1} \prec r_{2}\end{cases}
$$

Definition 17. Let $h_{\bar{H}_{1}}(x)$ and $h_{\bar{H}_{2}}(x)$ be two IVHFEs. The possibility degree of $h_{\bar{H}_{1}}(x) \geq h_{\bar{H}_{2}}(x)$ is defined as follows:

$$
\begin{aligned}
P & \left(h_{\bar{H}_{1}}(x) \succeq h_{\bar{H}_{2}}(x)\right) \\
& =\frac{1}{l_{\max }} \sum_{j=1}^{l_{\max }} f\left(h_{\bar{H}_{1}}{ }^{\sigma(j)}(x), h_{\bar{H}_{2}}{ }^{\sigma(j)}(x)\right) .
\end{aligned}
$$

Definition 18. Let $h_{\bar{H}_{1}}(x)$ and $h_{\bar{H}_{2}}(x)$ be two IVHFEs:

(1) If $P\left(h_{\bar{H}_{1}}(x) \succeq h_{\bar{H}_{2}}(x)\right)=0.5$, then IVHFE $h_{\bar{H}_{1}}(x)$ is quasi-equal to $h_{\bar{H}_{2}}(x)$, denoted by $h_{\bar{H}_{1}}(x) \approx h_{\bar{H}_{2}}(x)$.

(2) If $P\left(h_{\bar{H}_{1}}(x) \geq h_{\bar{H}_{2}}(x)\right)>0.5$, then IVHFE $h_{\bar{H}_{1}}(x)$ is more than $h_{\bar{H}_{2}}(x)$, denoted by $h_{\bar{H}_{1}}(x)>h_{\bar{H}_{2}}(x)$.

(3) If $P\left(h_{\bar{H}_{1}}(x) \geq h_{\bar{H}_{2}}(x)\right)<0.5$, then IVHFE $h_{\bar{H}_{1}}(x)$ is less than $h_{\bar{H}_{2}}(x)$, denoted by $h_{\bar{H}_{1}}(x) \prec h_{\bar{H}_{2}}(x)$.

(4) If $P\left(h_{\bar{H}_{1}}(x) \geq h_{\bar{H}_{2}}(x)\right) \geq 0.5$, then IVHFE $h_{\bar{H}_{1}}(x)$ is more than or equal to $h_{\bar{H}_{2}}(x)$, denoted by $h_{\bar{H}_{1}}(x) \geq$ $h_{\bar{H}_{2}}(x)$.

(5) If $P\left(h_{\bar{H}_{1}}(x) \geq h_{\bar{H}_{2}}(x)\right) \leq 0.5$, then IVHFE $h_{\bar{H}_{1}}(x)$ is less than or equal to $h_{\bar{H}_{2}}(x)$, denoted by $h_{\bar{H}_{1}}(x) \preceq$ $h_{\bar{H}_{2}}(x)$.

Example 19. We consider two IVHFEs given in Example 14. Since $l\left(h_{\bar{H}_{1}}(x)\right)>l\left(h_{\bar{H}_{2}}(x)\right)$, we extend $h_{\bar{H}_{2}}(x)=$ $\{[0.3,0.4],[0.3,0.4],[0.5,0.6]\}$ based on the pessimistic principle. By Definition 17, we have $P\left(h_{\bar{H}_{1}}(x) \geq h_{\bar{H}_{2}}(x)\right)=2 / 3$; then $h_{\bar{H}_{1}}(x)>h_{\bar{H}_{2}}(x)$.

Theorem 20. Let $h_{\bar{H}_{1}}(x)$ and $h_{\bar{H}_{2}}(x)$ be two IVHFEs. The following conclusions hold:

(1) $0 \leq P\left(h_{\bar{H}_{1}}(x) \geq h_{\bar{H}_{2}}(x)\right) \leq 1$. 
(2) $P\left(h_{\bar{H}_{1}}(x) \geq h_{\bar{H}_{2}}(x)\right)=1$ if and only if $h_{\bar{H}_{1}}{ }^{\sigma(j)}(x)>$ $h_{\bar{H}_{2}}{ }^{\sigma(j)}(x)$ for all $j=1,2, \ldots, l_{\text {max }}$.

(3) $P\left(h_{\bar{H}_{1}}(x) \geq h_{\bar{H}_{2}}(x)\right)=0$ if and only if $h_{\bar{H}_{1}}{ }^{\sigma(j)}(x) \prec$ $h_{\bar{H}_{2}}{ }^{\sigma(j)}(x)$ for all $j=1,2, \ldots, l_{\max }$.

Proof. (i) It is easy to prove $0 \leq P\left(h_{\bar{H}_{1}}(x) \geq h_{\bar{H}_{2}}(x)\right) \leq 1$ by Definition 17.

(ii) For necessity, if $P\left(h_{\bar{H}_{1}}(x) \geq h_{\bar{H}_{2}}(x)\right)=1$, then $f\left(h_{\bar{H}_{1}}{ }^{\sigma(j)}(x), h_{\bar{H}_{2}}{ }^{\sigma(j)}(x)\right)=1$ for all $j=1,2, \ldots, l_{\max }$ by Definitions 16 and 17. This implies that $h_{\bar{H}_{1}}{ }^{\sigma(j)}(x)>h_{\bar{H}_{2}}{ }^{\sigma(j)}(x)$ for all $j=1,2, \ldots, l_{\max }$.

For sufficiency, if $h_{\bar{H}_{1}}{ }^{\sigma(j)}(x)>h_{\bar{H}_{2}}{ }^{\sigma(j)}(x)$, then $f\left(h_{\bar{H}_{1}}{ }^{\sigma(j)}(x)\right.$, $\left.h_{\bar{H}_{2}}{ }^{\sigma(j)}(x)\right)=1$ for all $j=1,2, \ldots, l_{\max }$ by Definition 16 ; thus, $P\left(h_{\bar{H}_{1}}(x) \geq h_{\bar{H}_{2}}(x)\right)=1$ by Definition 17 .

(iii) Similar to (2), we can prove (3).

Remark 21. Formula (7) in Definition 17 can also be defined as follows:

$$
\begin{aligned}
P & \left(h_{\bar{H}_{1}}(x) \geq h_{\bar{H}_{2}}(x)\right) \\
& =\frac{1}{l_{\max }} \sum_{j=1}^{l_{\max }} P\left(h_{\bar{H}_{1}}{ }^{\sigma(j)}(x) \geq h_{\bar{H}_{2}}{ }^{\sigma(j)}(x)\right) .
\end{aligned}
$$

However, it does not satisfy Theorem 20. Example 22 can illustrate the point as follows.

Example 22. There are $h_{\bar{H}_{1}}(x)=\{[0.3,0.5],[0.3,0.6],[0.5$, $0.7]\}$ and $h_{\bar{H}_{2}}(x)=\{[0.3,0.4],[0.5,0.6]\}$. Based on the pessimistic principle, we extend $h_{\bar{H}_{2}}(x)=\{[0.3,0.4],[0.3,0.4]$, $[0.5,0.6]\}$, and then $P\left(h_{\bar{H}_{1}}(x) \geq h_{\bar{H}_{2}}(x)\right)=25 / 36 \neq 1$ by Formula (8) in Remark 21. There is a contradiction to Theorem 20, since $h_{\bar{H}_{1}}{ }^{\sigma(j)}(x)>h_{\bar{H}_{2}}{ }^{\sigma(j)}(x)$ for all $j=1,2,3$.

Definition 23. Let $\bar{H}_{1}$ and $\bar{H}_{2}$ be two interval-valued hesitant fuzzy sets on $X$ :

(1) If $h_{\bar{H}_{1}}(x) \preceq h_{\bar{H}_{2}}(x)$, for all $x \in X$, then $\bar{H}_{1}$ is an interval-valued hesitant fuzzy quasi subset of $\bar{H}_{2}$, denoted by $\bar{H}_{1} \subseteq \bar{H}_{2}$.

(2) If $h_{\bar{H}_{1}}(x) \approx h_{\bar{H}_{2}}(x)$, for all $x \in X$, then $\bar{H}_{1}$ is quasiequal to $\bar{H}_{2}$, denoted by $\bar{H}_{1} \approx \bar{H}_{2}$.

\section{Two Important Definitions of Interval-Valued Hesitant Fuzzy Soft Sets Based on the New Comparative Law}

In this section, we give definitions of the interval-valued hesitant fuzzy soft quasi subset and soft quasi equal according to Definition 23. Let $A, B \subseteq E$ and let $(\bar{F}, A)$ and $(\bar{G}, B)$ be two IVHFSSs over $U$.
TABLE 1: Tabular representation of $\left(\overline{F_{k}}, A_{k}\right)$.

\begin{tabular}{lcccc}
\hline & $u_{1}$ & $u_{2}$ & $\cdots$ & $u_{n}$ \\
\hline$e_{1}{ }^{k}$ & $\bar{h}_{\bar{F}_{k}\left(e_{1}{ }^{k}\right)}\left(u_{1}\right)$ & $\bar{h}_{\bar{F}_{k}\left(e_{1}{ }^{k}\right)}\left(u_{2}\right)$ & $\cdots$ & $\bar{h}_{\bar{F}_{k}\left(e_{1}{ }^{k}\right)}\left(u_{n}\right)$ \\
$e_{2}{ }^{k}$ & $\bar{h}_{\bar{F}_{k}\left(e_{2}{ }^{k}\right)}\left(u_{1}\right)$ & $\bar{h}_{\bar{F}_{k}\left(e_{2}{ }^{k}\right)}\left(u_{2}\right)$ & $\cdots$ & $\bar{h}_{\bar{F}_{k}\left(e_{2}{ }^{k}\right)}\left(u_{n}\right)$ \\
$\vdots$ & $\vdots$ & $\vdots$ & $\ddots$ & $\vdots$ \\
$e_{l\left(A_{k}\right)}{ }^{k}$ & $\bar{h}_{\bar{F}_{k}\left(e_{\left.l\left(A_{k}\right)^{k}\right)}\left(u_{1}\right)\right.}$ & $\bar{h}_{\bar{F}_{k}\left(e_{\left.l\left(A_{k}\right)^{k}\right)}\left(u_{2}\right)\right.}$ & $\cdots$ & $\bar{h}_{\bar{F}_{k}\left(e_{l\left(A_{k}\right)}{ }^{k}\right)}\left(u_{n}\right)$ \\
\hline
\end{tabular}

Definition 24. $(\bar{F}, A)$ is an interval-valued hesitant fuzzy soft quasi subset of $(\bar{G}, B)$, if the following two conditions are satisfied:

(1) $A \subseteq B$.

(2) $\bar{F}(e) \subseteq \bar{G}(e)$ for all $e \in A$. That is, $h_{\bar{F}(e)}(u) \preceq h_{\bar{G}(e)}(u)$, for all $e \in A$ and $u \in U$, denoted by $(\bar{F}, A) \bar{\subseteq}(\bar{G}, B)$.

Definition 25. $(\bar{F}, A)$ is soft quasi-equal to $(\bar{G}, B)$, if the following two conditions are satisfied:

(1) $A=B$.

(2) $\bar{F}(e) \approx \bar{G}(e)$ for all $e \in A$. That is, $h_{\bar{F}(e)}(u) \approx h_{\bar{G}(e)}(u)$, for all $e \in A$ and $u \in U$, denoted by $(\bar{F}, A) \cong(\bar{G}, B)$.

Theorem 26. $(\bar{F}, A) \cong(\bar{G}, B)$ if and only if $(\bar{F}, A) \subseteq(\bar{G}, B)$ and $(\bar{G}, B) \subseteq(\bar{F}, A)$.

Proof. For necessity, since $(\bar{F}, A) \cong(\bar{G}, B)$, we have $A=B$ and $h_{\bar{F}(e)}(u) \approx h_{\bar{G}(e)}(u)$ by Definition 25 . This implies that $A \subseteq B$ and $\bar{F}(e) \subseteq \bar{G}(e)$ for all $e \in A$. Analogously, $A \supseteq B$ and $\bar{F}(e) \supseteq$ $\bar{G}(e)$ for all $e \in A$. By Definition 24 , we have $(\bar{F}, A) \subseteq(\bar{G}, B)$ and $(\bar{G}, B) \subseteq(\bar{F}, A)$.

For sufficiency, since $(\bar{F}, A) \bar{\subseteq}(\bar{G}, B)$ and $(\bar{G}, B) \bar{\subseteq}(\bar{F}, A)$, we have $A \subseteq B$ and $\bar{F}(e) \subseteq \bar{G}(e)$ for all $e \in A$ by Definition 24 . Analogously, $A \supseteq B$ and $\bar{F}(e) \supseteq \bar{G}(e)$ for all $e \in A$. That is, $A=$ $B$ and $\bar{F}(e) \approx \bar{G}(e)$ for all $e \in A$. Therefore, $(\bar{F}, A) \cong(\bar{G}, B)$ by Definition 25.

\section{A New Method of Multiattribute Decision-Making Based on Interval-Valued Hesitant Fuzzy Soft Sets}

In the case of multiple attributes, the MADM is the decision problem to select the optimal alternative or rank the alternatives. Let $U=\left\{u_{1}, u_{2}, \ldots, u_{n}\right\}$ be the set of $n$ alternatives, let $E=\left\{e_{1}, e_{2}, \ldots, e_{t}\right\}$ be the set of $t$ attributes, and let $D=$ $\left\{d_{1}, d_{2}, \ldots, d_{s}\right\}$ be the set of $s$ decision-makers. The decisionmakers $d_{k} \in D(k=1,2, \ldots, s)$ evaluate $n$ alternatives from $A_{k}=\left\{e_{1}^{k}, e_{2}{ }^{k}, \ldots, e_{l\left(A_{k}\right)}{ }^{k}\right\}$, where $A_{k} \subseteq E$ and $l\left(A_{k}\right)$ represents the number of attributes in $A_{k}$. The attribute weight vector of $A_{k}$ is $\omega_{k}=\left(\omega_{1}{ }^{k}, \omega_{2}{ }^{k}, \ldots, \omega_{l\left(A_{k}\right)}{ }^{k}\right)^{T}$, where $\sum_{j=1}^{l\left(A_{k}\right)} \omega_{j}{ }^{k}=$ 1.

IVHFSSs $\left(\overline{F_{k}}, A_{k}\right)(k=1,2, \ldots, s)$ can be expressed by a tabular representation shown in Table 1 . Firstly, decisionmaker $d_{k}$ selects relevant attribute set $A_{k}$ according to 
his preference. Next, we collect the historical data of each attribute in the attribute set $E$ and use it as the basis for the evaluation of decision-maker. For example, economy factor is determined by market price, and workforce factor is determined by population, and so forth. Finally, $\bar{h}_{\bar{F}_{k}\left(e_{j}{ }^{k}\right)}\left(u_{i}\right)$ represents the IVHFE of the alternative $u_{i}$ satisfying the attribute $e_{j}{ }^{k}$ which is given by the decision-maker $d_{k}$, where $j=1,2, \ldots, l\left(A_{k}\right)$ and $i=1,2, \ldots, n$.

Definition 27. Suppose that $A=\left\{e_{1}, e_{2}, \ldots, e_{t}\right\}$ and $(\bar{F}, A)$ is an IVHFSS over $U$. A generalized comparison table (GCT) of $(\bar{F}, A)$ is a square table in which the number of rows and number of columns are equal. Both rows and columns are named by $U=\left\{u_{1}, u_{2}, \ldots, u_{n}\right\}$, and elements in GCT are denoted by $C_{i j}$. Its formula is shown as follows:

$$
\begin{aligned}
C_{i j}=\sum_{m=1}^{t} P\left(h_{\bar{F}_{e_{m}}}\left(u_{i}\right)\right. & \left.\geq h_{\bar{F}_{e_{m}}}\left(u_{j}\right)\right), \\
& i, j=1,2, \ldots, n ; m=1,2, \ldots, t
\end{aligned}
$$

where $t$ is the number of attributes.

Definition 28. Let $h_{\bar{H}}(x)=\left\{r=\left[r^{L}, r^{U}\right] \mid r \in h_{\bar{H}}(x)\right\}$ be an IVHFE and let $\lambda$ be a nonnegative real number; then a new multiplication operational law of IVHFEs is defined as follows:

$$
\lambda\left(h_{\bar{H}}(x)\right)=\left\{\left[\lambda r^{L}, \lambda r^{U}\right] \mid r \in h_{\bar{H}}(x)\right\}, \quad \lambda>0 .
$$

Obviously, it is different from Definition 7.

By the IVHFSSs based on the new comparative law of IVHFEs, we propose a new method of the MADM as follows.

\section{Algorithm 29.}

Step 1. Obtain the tabular representation of $\left(\overline{F_{k}}, A_{k}\right)$ by $\bar{F}_{k}\left(e_{j}{ }^{k}\right)=\left\{\bar{h}_{\bar{F}_{k}\left(e_{j}{ }^{k}\right)}\left(u_{i}\right) / u_{i} \mid u_{i} \in U\right\}\left(j=1,2, \ldots, l\left(A_{k}\right)\right)$ for all decision-makers $d_{k}(k=1,2, \ldots, s)$.

Step 2. Compute the weighted IVHFSS $\left(\bar{F}_{k}^{\prime}, A_{k}\right)$ for all $e_{j}{ }^{k} \in$ $A_{k}$ by ${\overline{F^{\prime}}}_{k}\left(e_{j}{ }^{k}\right)=\left\{\bar{h}_{\bar{F}_{k}\left(e_{j}{ }^{k}\right)}\left(u_{i}\right) / u_{i} \mid \bar{h}_{\bar{F}_{k}^{\prime}\left(e_{j}{ }^{k}\right)}\left(u_{i}\right)=\omega_{j}{ }^{k} \bar{h}_{\bar{F}_{k}\left(e_{j}{ }^{k}\right)}\right.$ $\left.\left(u_{i}\right), u_{i} \in U\right\}$.

Step 3. Compute $(\bar{F}, A)=\bar{\bigcup}_{k=1}^{s}\left(\bar{F}_{k}^{\prime}, A_{k}\right)$, where $A=\bigcup_{k=1}^{s}$ $A_{k}$.

Step 4. Calculate $C_{i j}$ according to Formula (9) and construct the GCT.

Step 5. Compute row sum $p_{i}=\sum_{j=1}^{n} C_{i j}$ and column sum $q_{j}=$ $\sum_{i=1}^{n} C_{i j}$ of the GCT, and calculate the score $S_{i}=p_{i}-q_{j}$ of alternative $u_{i}$.

Step 6. If the score $S_{i}$ is the maximum value, then $u_{i}$ can be used as the optimal alternative.

\section{Evaluate the Importance of the Major Components of the Well Drilling Mud Pumps}

A mud pump, an important part of the well drilling equipment, is a large reciprocating pump, which is used to circulate the mud on a drilling rig. A mud pump is mainly composed of four parts which are pump case, piston, bearing, and impeller. However, the four parts are also most likely to be damaged. Once one of these parts is damaged, the mud pump will not work properly and probably cause huge losses. Aiming at this problem, we will use Algorithm 29 to evaluate the importance of the four parts of the well drilling mud pump.

Now, we consider four parameters, namely, economic factor, reliability factor, service life factor, and maintenance factor, to evaluate the importance of the four parts. In order to make evaluation by three experts reasonably and comprehensively, we investigated the market price of the four parts in recent years for economic factor, collected the information feedback of the four parts for reliability factor, surveyed the durable years of the four parts for service life factor, and counted the maintenance time of the four parts for maintenance factor. Let $U=\left\{u_{1}, u_{2}, u_{3}, u_{4}\right\}$ be a set of the four parts, let $D=\left\{d_{1}, d_{2}, d_{3}\right\}$ be the set of the three experts, and let $E=\left\{e_{1}, e_{2}, e_{3}, e_{4}\right\}$ be the set of the four attributes.

Based on the evaluation of the three experts, we construct three tabular representations of $\left(\bar{F}_{k}, A_{k}\right)(k=1,2,3)$. The evaluations of experts are given as IVHFEs. The information is shown in Tables $2-4$.

The weight vector $\omega_{k}$ is given by three technical experts $d_{k}(k=1,2,3)$, where $\omega_{1}=(0.6,0.4)^{T}, \omega_{2}=(0.7,0.3)^{T}$, and $\omega_{3}=(0.4,0.6)^{T}$, respectively. Calculate the weighted IVHFSSs $\left(\bar{F}_{1}^{\prime}, A_{1}\right),\left(\bar{F}_{2}^{\prime}, A_{2}\right)$, and $\left(\bar{F}_{3}^{\prime}, A_{3}\right)$. Their representations are shown in Tables $5-7$.

Let $(\bar{F}, A)=\bar{\cup}_{k=1}^{3}\left(\bar{F}_{k}^{\prime}, A_{k}\right)$; then $A=A_{1} \cup A_{2} \cup A_{3}=$ $\left\{e_{1}, e_{2}, e_{3}, e_{4}\right\}$ and

$$
\begin{gathered}
\bar{F}\left(e_{1}\right)=\bar{F}_{1}^{\prime}\left(e_{1}\right)=\left\{\frac{\{[0.3,0.36],[0.24,0.48]\}}{u_{1}},\right. \\
\frac{\{[0.24,0.3],[0.24,0.42],[0.36,0.48]\}}{u_{2}}, \\
\frac{\{[0.3,0.48],[0.36,0.48]\}}{u_{3}}, \\
\left.\frac{\{[0.12,0.24],[0.3,0.36]\}}{u_{4}}\right\}, \\
\bar{F}\left(e_{2}\right)=\bar{F}_{2}^{\prime}\left(e_{2}\right) \cup \bar{F}_{3}^{\prime}\left(e_{2}\right) \\
=\left\{\frac{\{[0.28,0.35],[0.49,0.63]\}}{u_{1}},\right. \\
\frac{\{[0.07,0.21],[0.08,0.21],[0.14,0.35]\}}{u_{2}}, \\
\frac{\{[0.24,0.36],[0.28,0.36],[0.35,0.42]\}}{u_{3}}, \\
\left.\frac{\{[0.24,0.28],[0.28,0.32],[0.35,0.42]\}}{u_{4}}\right\}
\end{gathered}
$$


TABLE 2: Tabular representation of $\left(\bar{F}_{1}, A_{1}\right)$.

\begin{tabular}{ccccc}
\hline & $u_{1}$ & $u_{2}$ & $u_{3}$ & $u_{4}$ \\
\hline$e_{1}$ & $\{[0.5,0.6],[0.4,0.8]\}$ & $\{[0.4,0.5],[0.4,0.7],[0.6,0.8]\}$ & $\{[0.5,0.8],[0.6,0.8]\}$ & $\{[0.2,0.4],[0.5,0.6]\}$ \\
$e_{3}$ & $\{[0.1,0.2],[0.4,0.5],[0.7,0.8]\}$ & $\{[0.4,0.6],[0.5,0.6]\}$ & $\{[0.1,0.2],[0.2,0.3]\}$ & $\{[0.2,0.3],[0.2,0.4]\}$ \\
\hline
\end{tabular}

TABLE 3: Tabular representation of $\left(\bar{F}_{2}, A_{2}\right)$.

\begin{tabular}{ccccc} 
& $u_{1}$ & $u_{2}$ & $u_{3}$ & $u_{4}$ \\
\hline$e_{2}$ & $\{[0.4,0.5],[0.7,0.9]\}$ & $\{[0.1,0.3],[0.2,0.5]\}$ & $\{[0.2,0.5],[0.3,0.5],[0.5,0.6]\}$ & $\{[0.3,0.4],[0.5,0.6]\}$ \\
$e_{3}$ & $\{[0.6,0.8],[0.7,0.8]\}$ & $\{[0.3,0.7],[0.6,0.8]\}$ & $\{[0.3,0.5],[0.4,0.7]\}$ & $\{[0.3,0.6],[0.5,0.6]\}$ \\
\hline
\end{tabular}

TABLE 4: Tabular representation of $\left(\bar{F}_{3}, A_{3}\right)$.

\begin{tabular}{ccccc}
\hline & $u_{1}$ & $u_{2}$ & $u_{3}$ & $u_{4}$ \\
\hline$e_{2}$ & $\{[0.3,0.5],[0.4,0.7]\}$ & $\{[0.2,0.3],[0.1,0.5]\}$ & $\{[0.6,0.9],[0.7,0.9]\}$ & $\{[0.6,0.7],[0.7,0.8]\}$ \\
$e_{4}$ & $\{[0.1,0.3],[0.2,0.5],[0.4,0.6]\}$ & $\{[0.4,0.7],[0.5,0.8]\}$ & $\{[0.6,0.8],[0.7,0.8]\}$ & $\{[0.4,0.5],[0.5,0.7]\}$ \\
\hline
\end{tabular}

$$
\begin{aligned}
& \bar{F}\left(e_{3}\right)=\bar{F}_{1}^{\prime}\left(e_{3}\right) \cup \bar{F}_{2}^{\prime}\left(e_{3}\right) \\
& =\left\{\frac{\{[0.18,0.24],[0.21,0.24],[0.28,0.32]\}}{u_{1}},\right. \\
& \frac{\{[0.16,0.24],[0.18,0.24],[0.2,0.24]\}}{u_{2}}, \\
& \frac{\{[0.09,0.15],[0.12,0.21]\}}{u_{3}}, \\
& \left.\frac{\{[0.09,0.18],[0.15,0.18]\}}{u_{4}}\right\}, \\
& \bar{F}\left(e_{4}\right)=\bar{F}_{3}^{\prime}\left(e_{4}\right) \\
& =\left\{\frac{\{[0.06,0.18],[0.12,0.3],[0.24,0.36]\}}{u_{1}},\right. \\
& \frac{\{[0.24,0.42],[0.3,0.48]\}}{u_{2}}, \\
& \frac{\{[0.36,0.48],[0.42,0.48]\}}{u_{3}} \\
& \left.\frac{\{[0.24,0.3],[0.3,0.42]\}}{u_{4}}\right\} \text {. }
\end{aligned}
$$

According to Formula (9), we can calculate $C_{i j}(i, j=$ $1,2,3,4)$ and get the GCT of the IVHFSS $(\bar{F}, A)$ shown in Table 8.

From Table 8, we can get the row sum $p_{i}$, the column sum $q_{j}$, and the score $S_{i}$ of $u_{i}$ shown in Table 9 .
From Table 9, we have $S_{3}>S_{1}>S_{2}>S_{4}$; thus the part $u_{3}$, that is, bearing, is the most important component.

\section{Analysis and Discussion}

In the above MADM problem, it is very difficult for $d_{k}(k=$ $1,2,3)$ to determine his evaluation on every $e_{m}(m=$ $1,2,3,4)$ by a crisp number; thus some interval values in $[0,1]$ are used. Therefore, the existing methods [1521] are inapplicable to solve this problem. Furthermore, it is necessary to evaluate the mud pump from different aspects, since it is a complex system; thus comprehensive opinions by more than one decision-maker are needed, which implies that the existing method [14] considering a decision-maker is not appropriate to deal with this problem, too.

In addition, in the process of evaluating the importance of the four parts of the well drilling mud pump with Algorithm 29, we need to calculate $C_{i j}(i, j=1,2,3,4)$ by IVHFEs $h_{\bar{F}_{e_{m}}}\left(u_{i}\right)$ and $h_{\bar{F}_{e_{m}}}\left(u_{j}\right)(m=1,2,3,4)$ according to Formula (9). Therefore, the new comparative law given in Definition 17 is the base for computing $C_{i j}$.

\section{Conclusions}

Although Peng and Yang [14] have proposed IVHFSSs, the method they gave can only deal with MADM problems with a decision-maker. In most cases, it is necessary to synthesize opinions of more than one decision-maker for complex MADM problems. Based on IVHFSSs and the new comparative law, this paper presents a new method which can effectively solve MADM problems. We also apply this method to evaluate the importance of main parts of the well drilling mud pump, which can help engineers avoid the equipment failure as soon as possible. 
TABLE 5: Tabular representation of $\left(\bar{F}_{1}^{\prime}, A_{1}\right)$.

\begin{tabular}{|c|c|c|c|c|}
\hline & $u_{1}$ & $u_{2}$ & $u_{3}$ & $u_{4}$ \\
\hline$e_{1}$ & $\{[0.3,0.36],[0.24,0.48]\}$ & $\{[0.24,0.3],[0.24,0.42],[0.36,0.48]\}$ & $\{[0.3,0.48],[0.36,0.48]\}$ & $\{[0.12,0.24],[0.3,0.36]\}$ \\
\hline$e_{3}$ & $\{[0.04,0.08],[0.16,0.2],[0.28,0.32]\}$ & $\{[0.16,0.24],[0.2,0.24]\}$ & $\{[0.04,0.08],[0.08,0.12]\}$ & $\{[0.08,0.12],[0.08,0.16]\}$ \\
\hline
\end{tabular}

TABLE 6: Tabular representation of $\left(\bar{F}_{2}^{\prime}, A_{2}\right)$.

\begin{tabular}{ccccc}
\hline & $u_{1}$ & $u_{2}$ & $u_{3}$ & $u_{4}$ \\
\hline$e_{2}$ & $\{[0.28,0.35],[0.49,0.63]\}$ & $\{[0.07,0.21],[0.14,0.35]\}$ & $\{[0.14,0.35],[0.21,0.35],[0.35,0.42]\}$ & $\{[0.21,0.28],[0.35,0.42]\}$ \\
$e_{3}$ & $\{[0.18,0.24],[0.21,0.24]\}$ & $\{[0.09,0.21],[0.18,0.24]\}$ & $\{[0.09,0.15],[0.12,0.21]\}$ & $\{[0.09,0.18],[0.15,0.18]\}$ \\
\hline
\end{tabular}

TABLE 7: Tabular representation of $\left(\bar{F}_{3}^{\prime}, A_{3}\right)$.

\begin{tabular}{ccccc}
\hline & $u_{1}$ & $u_{2}$ & $u_{3}$ & $u_{4}$ \\
\hline$e_{2}$ & $\{[0.12,0.2],[0.16,0.28]\}$ & $\{[0.08,0.12],[0.04,0.2]\}$ & $\{[0.24,0.36],[0.28,0.36]\}$ & $\{[0.24,0.28],[0.28,0.32]\}$ \\
$e_{4}$ & $\{[0.06,0.18],[0.12,0.3],[0.24,0.36]\}$ & $\{[0.24,0.42],[0.3,0.48]\}$ & $\{[0.36,0.48],[0.42,0.48]\}$ & $\{[0.24,0.3],[0.3,0.42]\}$ \\
\hline
\end{tabular}

TABLE 8: The GCT of IVHFSS $(\bar{F}, A)$.

\begin{tabular}{ccccc}
\hline & $u_{1}$ & $u_{2}$ & $u_{3}$ & $u_{4}$ \\
\hline$u_{1}$ & 2 & $5 / 2$ & $5 / 3$ & $5 / 2$ \\
$u_{2}$ & $3 / 2$ & 2 & $7 / 6$ & 3 \\
$u_{3}$ & $7 / 3$ & $17 / 6$ & 2 & $37 / 12$ \\
$u_{4}$ & $3 / 2$ & 1 & $11 / 12$ & 2 \\
\hline
\end{tabular}

TABLE 9: The score $S_{i}$ of $u_{i}$.

\begin{tabular}{cccc}
\hline & $p_{i}$ & $q_{j}$ & $S_{i}$ \\
\hline$u_{1}$ & $26 / 3$ & $22 / 3$ & $4 / 3$ \\
$u_{2}$ & $23 / 3$ & $25 / 3$ & $-2 / 3$ \\
$u_{3}$ & $123 / 12$ & $23 / 4$ & $9 / 2$ \\
$u_{4}$ & $65 / 12$ & $127 / 12$ & $-31 / 6$ \\
\hline
\end{tabular}

\section{Conflicts of Interest}

The authors declare that there are no conflicts of interest regarding the publication of this paper.

\section{Acknowledgments}

This research is funded by the Open Fund of State Key Laboratory of Oil and Gas Reservoir Geology and Exploitation (Southwest Petroleum University) (no. PLN 1511) and the National Natural Science Foundation of China (no. 11401494).

\section{References}

[1] L. A. Zadeh, "Fuzzy sets," Information and Control, vol. 8, no. 3, pp. 338-353, 1965.

[2] K. T. Atanassov, "Intuitionistic fuzzy sets," Fuzzy Sets and Systems, vol. 20, no. 1, pp. 87-96, 1986.

[3] K. Atanassov and G. Gargov, "Interval valued intuitionistic fuzzy sets," Fuzzy Sets and Systems, vol. 31, no. 3, pp. 343-349, 1989.
[4] Z. Xu and M. Xia, "Distance and similarity measures for hesitant fuzzy sets," Information Sciences, vol. 181, no. 11, pp. 2128-2138, 2011.

[5] M. Xia and Z. Xu, "Hesitant fuzzy information aggregation in decision making," International Journal of Approximate Reasoning, vol. 52, no. 3, pp. 395-407, 2011.

[6] N. Chen, Z. S. Xu, and M. M. Xia, "Interval-valued hesitant preference relations and their applications to group decision making," Knowledge-Based Systems, vol. 37, pp. 528-540, 2013.

[7] D. Molodtsov, "Soft set theory-first results," Computers and Mathematics with Applications, vol. 37, no. 4-5, pp. 19-31, 1999.

[8] Z. Li, G. Wen, and N. Xie, "An approach to fuzzy soft sets in decision making based on grey relational analysis and Dempster-Shafer theory of evidence: an application in medical diagnosis," Artificial Intelligence in Medicine, vol. 64, no. 3, pp. 161-171, 2015.

[9] X. Q. Zhou, Soft set and hesitant fuzzy set theories with their application in decision making (in Chinese), Hunan University, 2014.

[10] P. K. Maji, R. Biswas, and A. R. Roy, "Fuzzy soft sets," Journal of Fuzzy Mathematics, vol. 9, no. 3, pp. 589-602, 2001.

[11] X. Yang, T. Y. Lin, J. Yang, Y. Li, and D. Yu, "Combination of interval-valued fuzzy set and soft set," Computers and Mathematics with Applications, vol. 58, no. 3, pp. 521-527, 2009.

[12] P. K. Maji, “Operations on intuitionistic fuzzy soft sets," Journal of Fuzzy Mathematics, vol. 20, no. 1, pp. 163-177, 2012.

[13] Y. Jiang, "Interval-valued intuitionistic fuzzy soft sets and their properties," Computers and Mathematics with Applications, vol. 60, no. 3, pp. 906-918, 2010.

[14] X. Peng and Y. Yang, "Interval-valued hesitant fuzzy soft sets and their application in decision making," Fundamenta Informaticae, vol. 141, no. 1, pp. 71-93, 2015.

[15] A. R. Roy and P. K. Maji, "A fuzzy soft set theoretic approach to decision making problems," Journal of Computational and Applied Mathematics, vol. 203, no. 2, pp. 412-418, 2007.

[16] Z. Kong, L. Gao, and L. Wang, "Comment on 'A fuzzy soft set theoretic approach to decision making problems," Journal of Computational and Applied Mathematics, vol. 223, no. 2, pp. 540-542, 2009. 
[17] F. Feng, Y. B. Jun, X. Liu, and L. Li, "An adjustable approach to fuzzy soft set based decision making," Journal of Computational and Applied Mathematics, vol. 234, no. 1, pp. 10-20, 2010.

[18] Y. Yang, X. Tan, and C. Meng, "The multi-fuzzy soft set and its application in decision making," Applied Mathematical Modelling. Simulation and Computation for Engineering and Environmental Systems, vol. 37, no. 7, pp. 4915-4923, 2013.

[19] Z. Xu and X. Zhang, "Hesitant fuzzy multi-attribute decision making based on TOPSIS with incomplete weight information," Knowledge-Based Systems, vol. 52, pp. 53-64, 2013.

[20] F. Q. Wang, X. Li, and X. H. Chen, "Hesitant fuzzy soft set and its applications in multicriteria decision making," Journal of Applied Mathematics, vol. 2014, Article ID 643785, 10 pages, 2014.

[21] J. Q. Wang, X. E. Li, and X. H. Chen, "Hesitant fuzzy soft sets with application in multicriteria group decision making problems," The Scientific World Journal, vol. 2015, Article ID 806983, 14 pages, 2015.

[22] Z. Xu, "Dependent uncertain ordered weighted aggregation operators," Information Fusion, vol. 9, no. 2, pp. 310-316, 2008.

[23] W. U. Jiang and D. S. Huang, "A review on ranking methods of interval numbers," Systems Engineering, vol. 22, no. 8, p. 1, 2004.

[24] Z. S. Xu and Q. L. Da, “The uncertain OWA operator," International Journal of Intelligent Systems, vol. 17, no. 6, pp. 569-575, 2002.

[25] F.-J. Gao, "Comprehensive ranking of possible degree and interval number," System Engineering Theory and Practice, vol. 33, no. 8, pp. 2033-2040, 2013 (Chinese). 


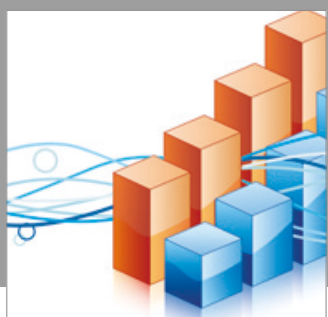

Advances in

Operations Research

vatersals

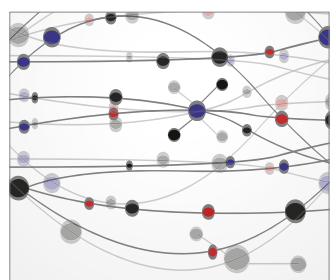

\section{The Scientific} World Journal
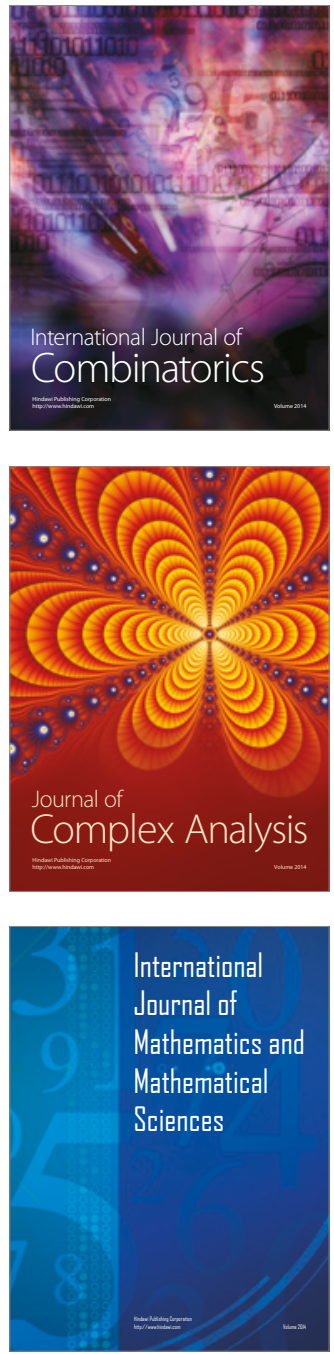
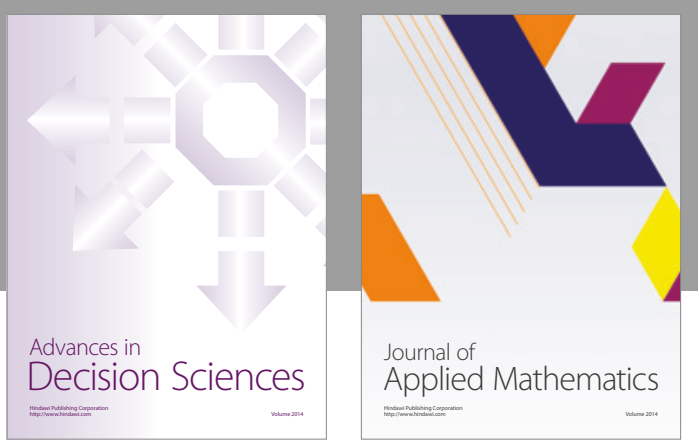

Algebra

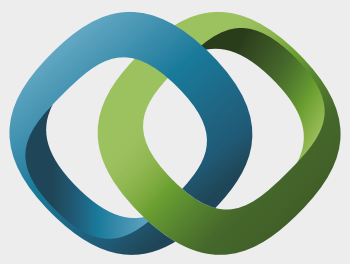

\section{Hindawi}

Submit your manuscripts at

https://www.hindawi.com
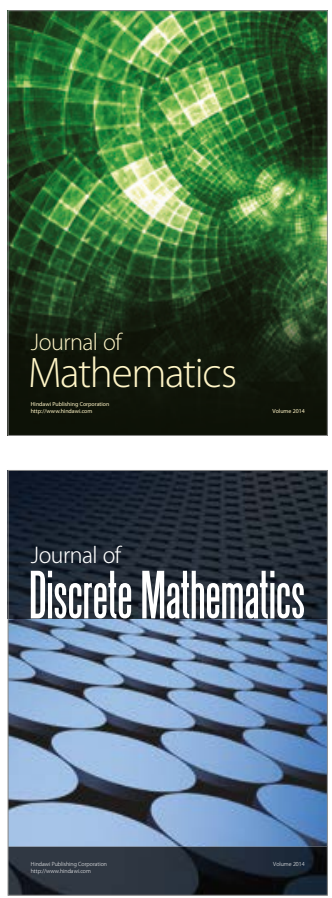

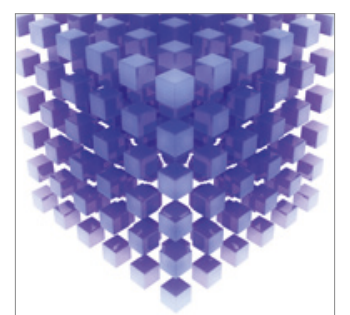

Mathematical Problems in Engineering
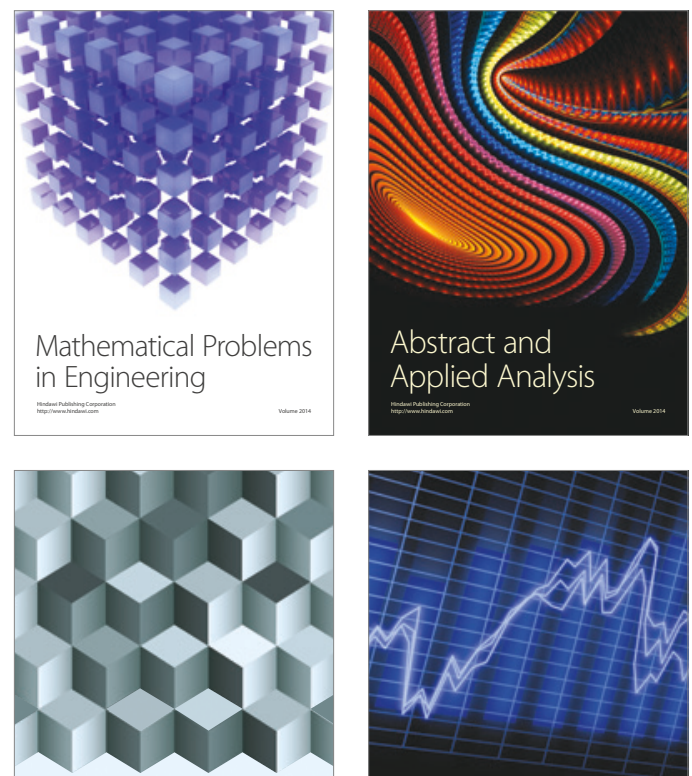

Journal of

Function Spaces

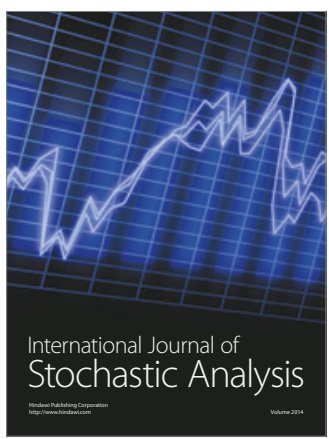

Probability and Statistics
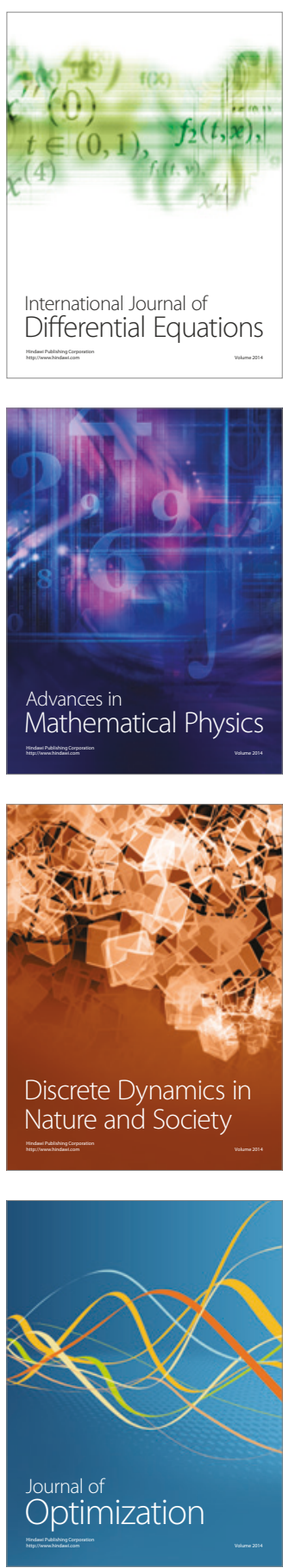